\section{Depression paths of least resistance}

\section{By Tim Fulmer, Senior Writer}

A pair of reports from U.S. and European researchers point to new targets in depression that could offer treatment alternatives to patients who are resistant or unresponsive to marketed antidepressants. ${ }^{1,2}$ One of the new targets, the protein P11, is already in the preclinical pipelines of Neurologix Inc. and Intra-Cellular Therapies Inc. for therapeutic and diagnostic applications, respectively.

Depression can be caused by low synaptic levels of serotonin in certain regions of the brain. Marketed antidepressants such as the selective serotonin reuptake inhibitors (SSRIs) block neurons from absorbing the neurotransmitter and increase its levels in the synapse.

The problem is that up to a third of depres-

\section{"In the near term we are looking at P11 more from the diagnostic than the therapeutic perspective." -Sharon Mates, Intra-Cellular Therapies Inc.}

To confirm that low levels of P11 in the nucleus accumbens were sufficient to cause depressive behavior, Greengard's team used the AAV vector to deliver p11 small hairpin RNA to the nucleus accumbens of wild-type mice. Indeed, shRNA-mediated knockdown of p11 increased depressive-like behaviors compared with those seen using control shRNA $(p<0.05)$.

Next, the researchers delivered a functional $p 11$ gene to the nucleus accumbens of mice using the same AAV vector.

In p11 knockout mice, the gene therapy significantly decreased multiple depressive-like behaviors compared with a control vector $(p<0.005)$.

Finally, to confirm the importance of P11 in humans, the researchers examined its expression in postmortem nucleus accumbens samples and found that P11 levels were significantly lower in depressed patients than in nondepressed controls $(p<0.05)$.

The group, which also included researchers from The University of Texas Southwestern Medical Center at Dallas, concluded that a $P 11$ gene therapy delivered to the nucleus accumbens "...may be useful as a treatment for select patients with major depression who prove refractory to other types of antidepressant treatment." The findings were published in Science Translational Medicine.

Kaplitt and colleagues at Neurologix, together sion patients don't respond to therapy or eventually develop some level of resistance. Thus, many researchers have begun to focus on pathways downstream of serotonin's action at the surface of the neuron.

\section{P11}

The first study is based on work by Paul Greengard, professor of molecular and cellular neuroscience at The Rockefeller University, and colleagues at the Karolinska Institute. The team previously reported that P11 (S100 calcium binding protein A10; S100A10), an intracellular protein involved in the trafficking of receptors to the cell surface, binds serotonin (1B) receptors on neurons, is aberrantly downregulated in rodent depression models and depression patients and is upregulated following antidepressant treatment. ${ }^{3-5}$

Based on those findings, the Greengard team hypothesized that increasing P11 levels in the brain might be a new avenue for depression therapeutics. To actually hit the target, Greengard turned to Michael Kaplitt, associate professor of neurological surgery and director of the laboratory of molecular neurosurgery at Weill Cornell Medical College and cofounder of neurology gene therapy company Neurologix.

The goal was to increase P11 levels in the nucleus accumbens of the brain by delivering the $P 11$ gene using an adenovirus-associated viral (AAV) vector. Neurologix already had proof of concept for the approach: in Phase I and Phase II trials, the company's glutamic acid decarboxylase $(G A D)$ gene delivered via AAV vectors was safe and effective in patients with Parkinson's disease (PD). ${ }^{6}$

The nucleus accumbens has been implicated in depression and other psychiatric disorders in animal models and patients. ${ }^{7,8}$ with Greengard's lab, are now collaborating with researchers at the National Institute of Mental Health to test the $P 11$ gene therapy in nonhuman primates.

"Moving forward, we are characterizing the safety and efficacy of the gene therapy by looking at behavioral and tissue-sample endpoints in the primates," Kaplitt told SciBX.

In parallel with the nonhuman primate studies, Kaplitt, Greengard and researchers from the Karolinska Institute and UT Southwestern are collaborating to develop a serum biomarker test that could be used to identify depression patients who might best benefit from $P 11$ gene therapy.

"At this point we see $P 11$ gene therapy as perhaps most appropriate for those depression patients who are nonresponders to the marketed oral antidepressants or those who develop resistance to the marketed therapies. Currently, two of the chief options potentially available to those patients are electroconvulsive shock therapy and deep brain stimulation," said Kaplitt.

Intra-Cellular, a company cofounded by Greengard, also is looking at the role of $\mathrm{P} 11$ in psychiatric disorders.

"In the near term we are looking at P11 more from the diagnostic than the therapeutic perspective," said Sharon Mates, chairman, CEO and cofounder of Intra-Cellular. "We want to better understand how P11 levels might serve as a biomarker for responsiveness to antidepressants."

In the longer term Mates said the company is "interested in developing small molecule compounds that could target P11 levels. Those compounds could either stand alone or be used together with P11 gene therapy." 
Figure 1. Targeting MKP1 in depression. Research published in Nature Medicine by a Yale School of Medicine team suggests that inhibiting dual specificity phosphatase 1 (DUSP1; MKP1) in neurons could help treat depression.

In neurons, extracellular growth factors bind receptors on the cell surface [a], triggering a phosphorylation cascade involving the kinases Ras, v-raf-1 murine leukemia (RAF), MAP kinase kinase (MEK) and MAP kinase (MAPK; ERK) [b]. The cascade ultimately activates transcription factors in the nucleus [c], triggering the transcription of multiple genes that promote cell growth and neural plasticity, which is thought to be essential for proper functioning of neurons.

In depression, MKP1, an inhibitory phosphatase that normally acts as a brake on the phosphorylation cascade [d], is aberrantly overexpressed, leading to less cell growth and plasticity, which can contribute to depression, anxiety and perhaps other psychiatric disorders.

The latest findings suggest that inhibiting MKP1 in depression could increase neurotrophic MEK and ERK signaling and improve psychiatric function.

According to Mates, "Intra-Cellular holds an exclusive license to IP from Rockefeller covering the role of P11 in depression and other psychiatric disorders." She declined to disclose further details.

Meanwhile, Neurologix has exclusively licensed a patent application from Cornell that specifically covers the use of $P 11$ gene therapy to treat depression and other psychiatric disorders. Kaplitt declined to comment on the fact that Intra-Cellular also holds a patent on P11 in psychiatric disorders.

Based on what has been disclosed by the two companies, it is not clear to what extent the claims of the two patents overlap, if at all. Nonetheless, both Kaplitt and Mates told SciBX they were open to the possibility of the two companies collaborating in the future on developing P11-targeting therapeutics.

Intra-Cellular's lead compound, ITI-007, a dual serotonin (5- $\left.\mathrm{HT}_{2 \mathrm{~A}}\right)$ receptor antagonist and dopamine receptor phosphoprotein modulator (DPPM), is in Phase II testing to treat schizophrenia.

Neurologix's AAV-GAD (NLX-P101), a GAD gene therapy delivered using an AAV vector, has completed a Phase II trial to treat moderate to advanced $\mathrm{PD}$.

\section{MKP1}

In the second study, a team at the Yale School of Medicine searched for new targets in depression using a genomewide analysis of postmortem hippocampal tissue samples from 21 depression patients and 18 healthy matched controls.

One of the most strongly dysregulated proteins in the hippocampus turned out to be dual specificity phosphatase 1 (DUSP1; MKP1). Expression of the enzyme was more than doubled in two different regions of hippocampal tissue from depression patients compared with in those regions in controls.

MKP1 is a negative regulator of the MAP kinase (MAPK; ERK) signaling pathway, which promotes growth in neurons and other cell types (see Figure 1, “Targeting MKP1 in depression”). Thus, the researchers

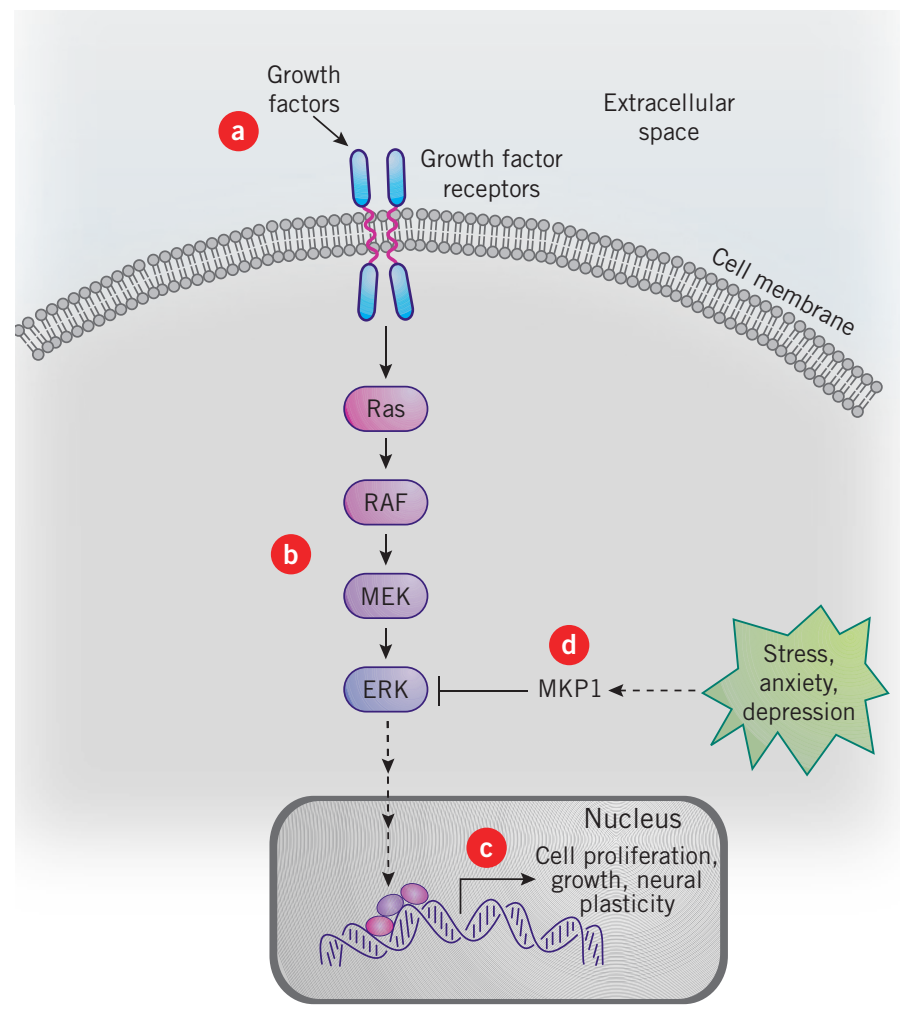

hypothesized that aberrantly increased MKP1 levels in the brain led to lower levels of ERK signaling and loss of neuronal growth, which could contribute to depression and other psychiatric disorders.

To test that hypothesis, a group led by Ronald Duman, professor of psychiatry and pharmacology at Yale, first looked for an association between high MKP1 levels and stress in rodents. In rats subjected to depressioninducing chronic stress, Mkp1 mRNA levels were indeed greater than nonstressed baseline levels in multiple regions of the hippocampus.

The researchers next asked whether high hippocampal MKP1 levels were sufficient to cause depressive-like behavior. They used AAV vectors to deliver the $M k p 1$ gene to regions of the hippocampus in mice and found that high Mkp1 levels indeed led to multiple depressive-like behaviors compared with delivery of control vector.

Finally, they asked whether knocking out $M k p 1$ could decrease depressive behavior in mice. Mkp1 knockout mice showed fewer depressed responses to stress than wild-type controls.

The authors wrote that pharmacological blockade of MKP1 could produce "a resilient or antidepressant response to stress, or possibly an enhanced response to other classes of antidepressants."

Results were published in Nature Medicine.

Corresponding author Duman told SciBX that he wants to continue to map out the mechanism and pathways that mediate the effects of high MKP1 levels in depression.

In the longer term, Duman is considering developing inhibitors of MKP1. That could be a challenging task, he noted, because "it's been historically difficult to develop inhibitors of phosphatases like MKP1. Part of the reason is that catalytic sites across different phosphatases are generally very similar, which can make it difficult to design selective phosphatase 


\section{ANALYSIS}

inhibitors that have a good side-effect profile. Nonetheless, we are in preliminary discussions with some undisclosed labs about the potential of designing MKP1 inhibitors."

Duman said the paper's findings are not patented.

Fulmer, T. SciBX 3(43); doi:10.1038/scibx.2010.1284

Published online Nov. 4, 2010

\section{REFERENCES}

1. Alexander, B. et al. Sci. Transl. Med.; published online Oct. 20, 2010; doi:10.1126/scitranslmed.3001079

Contact: Michael G. Kaplitt, Weill Cornell Medical College,

New York, N.Y.

e-mail: mik2002@med.cornell.edu

2. Duric, V. et al. Nat. Med.; published online Oct. 17, 2010;

doi:10.1038/nm.2219

Contact: Ronald S. Duman, Yale University, New Haven, Conn. e-mail: ronald.duman@yale.edu

3. Svenningsson, P. et al. Science 311, 77-80 (2006)

4. Svenningsson, P. et al. J. Neurosci. 27, 4201-4209 (2007)

5. Warner-Schmidt, J.L. et al. J. Neurosci. 29, 1937-1946 (2009)

6. Kaplitt, M.G. et al. Lancet 369, 2097-2105 (2007)

7. Nestler, E.J. \& Carlezon, W.A. Jr. Biol. Psychiatry 59, 1151-1159 (2006)

8. Zhang, X. et al. Proc. Natl. Acad. Sci. USA 105, 2163-2168 (2008)

COMPANIES AND INSTITUTIONS MENTIONED

Intra-Cellular Therapies Inc., New York, N.Y.

Karolinska Institute, Stockholm, Sweden

Neurologix Inc. (OTCBB:NRGX), Fort Lee, N.J.

National Institute of Mental Health, Bethesda, Md.

The Rockefeller University, New York, N.Y.

The University of Texas Southwestern Medical Center at Dallas,

Dallas, Texas

Weill Cornell Medical College, New York, N.Y.

Yale School of Medicine, New Haven, Conn. 